\title{
Situación General de la Industria de la Construcción de Bajo Bordo
}

\author{
General Situation of the Shipbuilding Board low
}

Alexander Chui ${ }^{1}$, Karla Cornejo ${ }^{1}$, Magali Gamero', Vanessa Rios ${ }^{1}$,
Maria Laura Valdivia' ${ }^{1}$, Jorge Benzaquén de las Casas $^{1}$

\begin{abstract}
RESUMEN
Objetivo: Determinar las condiciones en que se encuentra la industria de construcción naval de bajo bordo en el Perú, relacionado con su contexto latinoamericano. Métodos: Se estableció un protocolo de actuación a base entrevistas, cuestionarios y recopilación de datos con el fin de conocer los principales indicadores de la dinámica funcional e industria de construcción naval de bajo bordo. Resultados: El Perú ha crecido notablemente en las últimas dos décadas como consecuencia de la apertura comercial y el incremento de inversionistas, originando la dinamización de la economía y convirtiendo al país en uno de los más atractivos de la región. Su ubicación geoestratégica en la costa oeste del Pacífico sur, otorga al Perú la condición de hub natural, siendo oportunidad para que el puerto del Callao se convierta en el puerto hub de Sudamérica, facilitando el comercio entre países del Asia y América Latina, apoyado en la firma de tratados de libre comercio con dichos países. Así mismo, dada la extensa costa que posee el Perú, la industria de la construcción naval cobra importancia en el desarrollo económico sostenido, otorgando valor agregado a la producción nacional, generando mano de obra altamente calificada y propiciando el desarrollo de industrias relacionadas. Conclusiones: El Perú se encuentra ubicado en la costa oeste del Pacífico Sur y posee una larga costa que le otorga una ventaja comparativa en relación a otros países de Sudamérica. El crecimiento a nivel mundial del comercio ha generado el crecimiento del tráfico marítimo mundial de naves de alto bordo lo que genera el desarrollo de la industria de construcción naval de bajo bordo, pues son estas embarcaciones que brindan la asistencia requerida a las embarcaciones de alto bordo. La integración Perú - Brasil, la consolidación del Callao como puerto hub junto con las concesiones portuarias y de hidrovías y las potenciales oportunidades del sector pesquero permitirán la reactivación de la industria naval de bajo bordo.
\end{abstract}

Palabras clave: Planeamiento estratégico, industria naval de bajo bordo.

\begin{abstract}
Objective: To determine the conditions under which the shipbuilding industry in Peru on board, related to its Latin American context is. Methods: a protocol interviews, questionnaires and data collection in order to meet the main indicators of the functional dynamics and shipbuilding industry on board was established based. Results: Peru has grown significantly in the last two decades as a result of trade liberalization and increased investors, resulting in the revitalization of the economy and making the country one of the most attractive in the region. Its geostrategic location on the west coast of the South Pacific, grants to Peru's status as a natural hub, with opportunity for the port of Callao will become the hub port in South America, facilitating trade between countries of Asia and Latin America, supported by the signing of free trade agreements with those countries. Also, given the extensive coastline which owns Peru, the shipbuilding industry has become important in sustained economic development, providing added value to domestic production, generating highly skilled labor and promoting the development of related industries. Conclusions: Peru is located on the west coast of the South Pacific and has a long coastline which gives it a comparative advantage over other countries in South America. The worldwide growth of trade has generated growth in world seaborne trade ships high board which generates the development of shipbuilding industry low board, as are these vessels that provide the assistance required to craft high board. Integration Peru - Brazil, the consolidation of Callao as a hub port with port concessions and waterways and the potential opportunities in the fisheries sector will allow the reactivation of the naval industry on board.
\end{abstract}

Keywords: Strategic Planning, naval industry on board.

${ }^{1}$ Pontificia Universidad Católica del Perú. Lima - Perú 


\section{INTRODUCCIÓN}

La industria de la construcción naval es una industria líder en grandes países que cuentan con una ubicación geográfica estratégica marítima. Es el caso del Perú que tiene una posición privilegiada en América del Sur y cuenta con una larga franja costera que limita con el océano Pacífico. Por la dimensión de sus proyectos, esta industria posee un alto valor agregado, por lo tanto la magnitud de la inversión es elevada requiriendo procesos de producción con altos estándares de calificación internacional y un uso intensivo de mano de obra calificada.

Para situar a la industria de construcción naval de bajo bordo en el Perú se deberá analizar previamente aspectos relacionados al tráfico marítimo mundial en relación al tráfico en el Perú, el cabotaje marítimo y fluvial, el desarrollo del sector pesquero y la influencia de otras industrias relacionadas. En el periodo 19902010 el tráfico marítimo mundial se duplicó pasando de 4,008 millones de toneladas a 8,408 millones.

El crecimiento medio anual durante las tres últimas décadas ha sido de $3.1 \%$, lo que significa que en el 2020 el tráfico marítimo estimado sería de 11,500 millones de toneladas y en el 2031 alrededor de 16,010 millones de toneladas (APN, 2012). El crecimiento del tráfico marítimo de contenedores se debe al incremento en la demanda de bienes de consumo en regiones en desarrollo, a la organización de las compañías navieras (tráficos de trasbordo y vacíos), al sistema productivo mundial que supone el transporte de componentes y semielaborados, y a la contenedorización de algunos productos que tradicionalmente se transportaban a granel como productos forestales y algunos minerales.

La tendencia mundial de construcción de embarcaciones de alto bordo por la demanda antes señalada ha originado embarcaciones de dimensiones cada vez más grandes como los contenedores Panamax o Superpanamax, estas embarcaciones por sus dimensiones requieren del apoyo de embarcaciones menores de bajo bordo para su arribo, estadía y partida de los principales puertos mundiales. Por ende, el desarrollo de la industria de construcción naval de alto bordo tiene influencia directa en el crecimiento de la industria naval de construcción de embarcaciones de bajo bordo.

Si bien la industria de la construcción naval de bajo bordo no brinda altos márgenes de rentabilidad (no mayor al $8 \%$ ), la relación que se origina con industrias conexas y el impacto que esto produce en la economía, constituye un factor estratégico y de gran importancia para el desarrollo del país donde se desarrolla . A nivel de Sudamérica, el intercambio comercial internacional es de gran relevancia para el sector marítimo y portuario fomentando a la industria de construcción naval de bajo bordo, a través de la mayor prestación de servicios de transporte y logística de sus embarcaciones, siendo considerada como una de las industrias más importantes y con gran potencial de desarrollo para el crecimiento sostenido de la región (Sánchez, 2004). Los países de la costa oeste de América del Sur que poseen astilleros constructores estatales y privados son: Ecuador, Perú y Chile.

Este último es el país más competitivo de Sudamérica en la industria de la construcción naval de bajo bordo y cuenta con el Astillero Maestranzas de la Armada (ASMAR S.A.) y Ascón.

En el caso del Ecuador destacan los astilleros: Astilleros Navales del Ecuador y ASTINAVES. Perú es el segundo país más competitivo con una industria reconocida internacionalmente por su diseño y experiencia. La industria de construcción naval de bajo bordo ecuatoriana no se encuentra desarrollada y produce pocas embarcaciones.

El tráfico marítimo en el Perú, a pesar de la crisis mundial, ha presentado un mayor crecimiento debido a dos factores fundamentales: (a) la expansión económica sostenida de la economía peruana en la última década, y (b) la dinamización del comercio internacional por la firma de tratados de libre comercio, en especial con: (a) EEUU, (b) China, (c) Japón, (d) Corea del Sur, y (e) la Comunidad Europea; y por la firma del acuerdo de integración con Brasil. Para acompañar este ritmo de crecimiento económico se requiere de un alto nivel de competitividad, y este viene respaldado por la infraestructura que conecta al país internamente y externamente.

Es así que el Perú posee ventajas comparativas para convertirse en el nexo del movimiento de carga entre los países de América del Sur y los países que integran la cuenca Asia Pacífico, convirtiendo al Perú en el de mayor perspectiva a nivel mundial, por lo que es indispensable recortar la brecha en infraestructura que tiene el país para lograr la reactivación del sector naval.

En función a la ubicación estratégica del Perú, las oportunidades comerciales con las principales economías y las condiciones internas para convertirse en la principal plataforma de comercio en la región, se hace necesario contar con una flota mercante nacional. En el año 1991 se eliminaron todos los incentivos para la marina mercante nacional mediante Decreto $N^{\circ} 644$ adoptándose una política liberal para el transporte marítimo. Actualmente la mayor parte del tráfico marítimo internacional lo realizan 24 empresas con naves de bandera extranjera, sólo una empresa privada con embarcaciones de bandera peruana realiza el transporte internacional.

El Perú deja de percibir divisas por concepto de fletes con un efecto considerable en la balanza de pagos, los fletes son cobrados por empresas extranjeras (IEHMP, 2012). La Ley de Reactivación y Promoción de la Marina Mercante Nacional, Ley 28583 (Congreso de la República, 2005) fue promulgada por 
el gobierno de Alan García con el objeto de incentivar la competitividad de la industria naval en los mercados mundiales de transporte acuático.

Destaca la promoción de la construcción naval, que incluye a empresas públicas autorizadas, la exoneración del impuesto a las ventas y selectivo al consumo por compra de combustibles para buques de bandera nacional, la exigencia de que por lo menos $51 \%$ del capital de la empresa sea de propiedad nacional, la exclusividad del cabotaje para buques de bandera peruana, y en el caso de transporte de hidrocarburos, hasta en un $25 \%$ lo realizará los buques de la Marina, por razones de defensa nacional, el fomento de la Marina Mercante fluvial en la Amazonía peruana y la exoneración del impuesto general a las ventas y el impuesto selectivo al consumo a los servicios brindados a embarcaciones nacionales por la industria de construcción naval. Es decir, mediante esta norma se brinda mayor proteccionismo favoreciendo a la industria nacional y se pretende reactivar a la flota mercante nacional.

El puerto del Callao es el puerto más importante del país, octavo en América Latina y el Caribe, y actualmente se ha posicionado como el primer puerto de transbordo de contenedores en la costa oeste de Sudamérica, con un crecimiento de movimiento portuario de contenedores superior al $20 \%$ entre el año 2010 y el 2011. Este puerto es el punto final del eje de integración con Brasil mediante el proyecto "Iniciativa para la integración de la infraestructura regional Sudamericana" (IIRSA). El Callao podría convertirse en primer puerto hub de la región, en este sentido las concesiones realizadas en este puerto han sido progresivas.

Desde junio de 2010 el consorcio DP World Callao tiene a su cargo el muelle sur que se encuentra equipado para movilizar 850,000 TEUs al año. La segunda empresa es APM Terminals que opera el terminal norte multipropósito del puerto del Callao movilizando 2.9 millones de TEUs al año; adicionalmente se invertirá US\$120 millones en la construcción del Terminal de embarque de concentrados de minerales del T.P. del Callao. Estas inversiones que culminarán en el 2015 han reducido significativamente los tiempos de espera de embarque y desembarque de carga de las embarcaciones que se traduce en menores costos de operación y logística para importadores y sobre todo para exportadores (Advanced Logistics Group, 2011).

\section{MATERIALES Y MÉTODOS}

De acuerdo al reporte estadístico de la Autoridad Portuaria Nacional - APN (2012) y tal como se observa en la Tabla 1, el puerto del Callao en sus dos terminales, DP World Callao y APM Terminal Callao, ha recibido 3,299 naves de alto bordo durante el año 2012. En la Figura 2 se visualiza la participación de los tipos de naves recibidas: (a) $42.9 \%$ de portacontenedores, (b) $23.6 \%$ de carga general, (c) $20 \%$ de buques tanque, y (d) $13.4 \%$ de graneleros. De acuerdo al Reglamento de recepción y despacho de naves en los puertos de la República del Perú (Presidencia de la República del Perú, 2005), las embarcaciones de alto bordo que llegan al puerto del Callao requieren del apoyo de remolcadores y barcazas con funciones de: (a) abastecimiento de combustible y provisiones, (b) mantenimiento, y (c) descarga de mercaderías.

Tabla 1. Recibido en el Callao - Año 2012

\begin{tabular}{lcc}
\hline Tipo de Nave & Unidades & $\begin{array}{c}\text { Participación } \\
(\%)\end{array}$ \\
\hline Portacontenedores & 1,416 & 42.92 \\
Carga General & 780 & 23.64 \\
Tanques & 660 & 2.01 \\
Graneleros & 443 & 13.43 \\
\hline Total & 3,799 & 100.00 \\
\hline
\end{tabular}

Adaptado de "Reporte Anual 2012", por Autoridad Portuaria Nacional (APN), 2012.
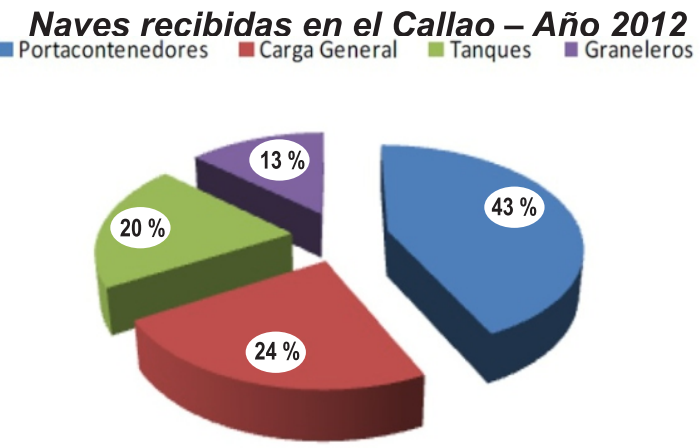

Figura 1. Participación de Naves recibidas en el Callao-Año 2012.

Adaptado de Reporte Anual 2012 por Autoridad Portuaridad Nacional APN, 2012.

A pesar del extenso litoral marítimo peruano y el potencial actual y futuro de la actividad de cabotaje, su desarrollo es muy limitado. Entre las dificultades para su desarrollo se mencionan: (a) conciencia marítima inexistente, (b) poco convencimiento sobre la utilidad del cabotaje, (c) ausencia de desarrollo portuario e infraestructura, (d) excesivas regulaciones que generan altos costos, (e) adecuación del transporte multimodal, (f) ausencia de políticas de transporte de cabotaje, y (g) preferencia por el uso de transporte terrestre (IEHMP, 2012).

En relación al desarrollo portuario, actualmente el Perú cuenta con ocho puertos de atraque directo en la costa, de los cuales siete movilizan tráfico de gráneles líquidos (petróleos y derivados) y están a cargo del Consorcio Terminales del grupo Graña y Montero. 
Los puertos de Paita, Chimbote, Ilo y Matarani tienen posibilidades de competencia interportuaria en carga suelta y contenedorizada, sin embargo necesitan tres condiciones (a) convertirse en centro de tráfico, (b) concentrar operadores, y (c) mejorar telecomunicaciones. La mayoría no cuenta con la infraestructura suficiente para incentivar el cabotaje marítimo, por lo tanto los costos portuarios son muy elevados restando competitividad al tránsito. Para el desarrollo de la actividad de cabotaje marítimo es indispensable la optimización y eficiencia de servicios brindados en puerto, especialmente los referentes a carga y descarga de embarcaciones. En este sentido APN ha iniciado la concesión de puertos para dotarlos del equipamiento necesario para la carga y descarga de mercancías, dragado y las facilidades para el ingreso y salida de los buques de tal modo que se incentive la actividad de cabotaje marítimo.

El transporte de cabotaje marítimo se encuentra limitado a carga de combustible, no se traslada otro tipo de carga porque no ofrece la competitividad que ofrece el transporte de carga por vía terrestre. Será necesaria la eficiencia en los procesos portuarios y el uso de transporte multimodal por una misma operadora para reducir costos que permitan la ampliación del sistema de transporte nacional que tenga como consecuencia una mayor demanda de embarcaciones de bajo bordo.

La actividad de cabotaje es de vital importancia en el sistema de transporte y comunicación fluvial. La concesión de hidrovías en la Amazonía mejorará la navegabilidad de los ríos Huallaga, Ucayali, Marañón y Amazonas, con una longitud de 2,269 kilómetros, siendo principal vía de circulación. Pro Inversión tiene programado para el 2013 la concesión de hidrovías que se iniciarán en el 2014 y culminarán en el 2016 con el apoyo de la inversión estatal conjunta del gobierno de Brasil y Perú (Agencia Peruana de Noticias, 2012). En este sentido el acuerdo de integración con Brasil IIRSA tiene como objetivo incrementar el comercio, desarrollando las regiones aisladas de la Amazonia, es el impulsador para incrementar y mejorar las hidrovías para hacer navegables los ríos de la selva durante los 12 meses del año (IIRSA, 2012).

De los cuatro ejes de IIRSA, toma relevancia para la industria naval de la construcción de embarcaciones de bajo bordo el Eje del Amazonas (Ver Figura 2) que combina el transporte intermodal carreteras hidrovías situado en el eje vial Norte. Este eje tiene como fin la integración con Brasil e interconexión de los puertos de la zona norte del país, donde el puerto de Paita ya se encuentra concesionado a la empresa Terminales Portuarios Euro Andinos, en el marco de las concesiones realizadas por APN. El desarrollo del transporte multimodal en el Eje Amazonas norte Paita - Yurimaguas contempla un corredor vial, modernización de los puertos Paita y Yurimaguas que será un punto de intercambio entre las ciudades de Chiclayo, Piura e lquitos. El concesionario es el Consorcio Portuario Yurimaguas de inversionistas peruano ecuatorianos, el proyecto requiere una inversión de US\$43,7 millones a realizarse durante los siguientes cinco años, el puerto contará con grúas pórtico y el dragado suficiente para que lleguen y partan buques desde y hacia Brasil, y de aquí sigan su camino al Asia (Diario Gestión , 2011).

Las concesiones de hidrovías, que se realizarán en los próximos años y las concesiones de puertos en la Amazonía a realizarse por APN dinamizarán las actividades de cabotaje fluvial, para transporte de carga de mercancías entre puertos regionales y desde Brasil hacia la costa peruana. Es así que la utilización de embarcaciones de bajo bordo se incrementará y la industria debe estar preparada para responder competitivamente ante el incremento de la demanda.

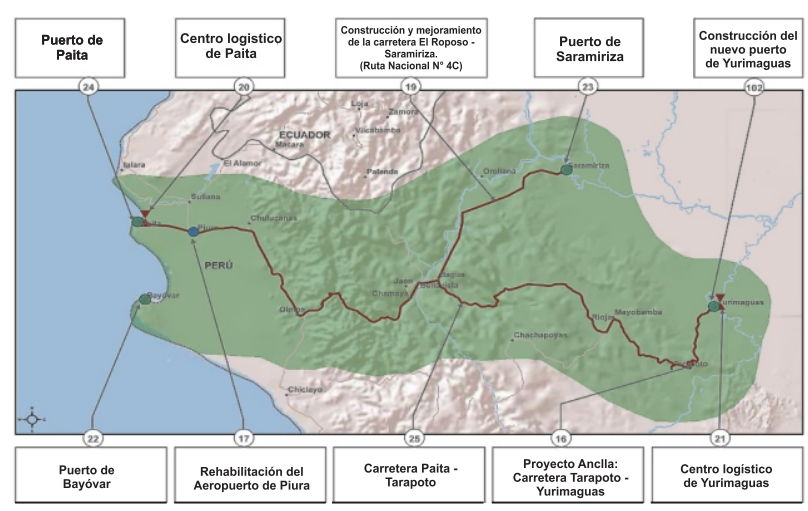

Figura 2. Eje IIRSA Amazónica.

Tomado de la "Ficha técnica del proyecto: Eje de Amazonas.\# por V. Gutierrez, 2012. IIRSA

Durante la década comprendida entre 1970 y 1980 era el sector pesquero uno de los clientes más importante de la industria de construcción naval; hoy en día es este sector el que demanda mayor cantidad de embarcaciones de segundo uso y a la fecha sólo se realizan modificaciones para su operatividad. Las nuevas condiciones para la captura del atún, podrían convertirse en una oportunidad de desarrollo de la industria naval de construcción de embarcaciones de bajo bordo.

La Comisión Interamericana del Atún Tropical (CIAT) ha otorgado al Perú un incremento de la cuota del atún de 3,195 metros cúbicos a 8,195 metros cúbicos, lo que podría demandar el uso de entre 13 a 15 embarcaciones peruanas como un inicio para desarrollar una flota atunera que permitirá realizar la pesca del atún y destinarla al consumo y alimentación de la población. La mayor asignación de la cuota de pesca de atún no sólo es para el Perú sino para otros países como el Ecuador que es líder de la actividad extractiva de este recurso a nivel regional y puede requerir un mayor número de embarcaciones para su flota (Andina, 2012).

La industria de la construcción naval en el Perú está conformada por cuatro organizaciones: (a) SIMA Perú, empresa estatal de derecho privado dentro del ámbito del ministerio de defensa, cuenta con tres 
astilleros, dos de ellos ubicados en la costa y uno ubicado en la Amazonía; (b) Construcciones Maggiolo S.A., astillero privado familiar ubicado en el Callao; (c) TASA, astillero privado ubicado en la costa norte; y (d) Henry E.I.R.L., astillero privado ubicado en la Amazonía. Estos astilleros brindan servicios de construcción y reparación naval de bajo bordo, centrándose principalmente en la construcción de embarcaciones remolcadoras, barcazas y pesqueras (SIMA, 2012). En la actualidad, SIMA se constituye como el principal representante de la industria de la construcción naval de bajo bordo nacional.

La industria de construcción naval en el Perú se encuentra en una etapa de crecimiento y a pesar de ser una industria de alto valor agregado, poseer la capacidad de espacios necesaria para desarrollarse y contar con la experiencia de muchos años de trayectoria, tiene algunas deficiencias: (a) la infraestructura no es moderna, (b) las líneas de producción llegan a tener una antigüedad de casi cuatro décadas, (c) no hay inversión en investigación y desarrollo, (d) el personal calificado es escaso, (e) la industria no cuenta con estrategias de integración con proveedores, (f) no hay políticas que impulsen la publicidad a nivel nacional e internacional, (g) la participación del estado es casi nula, y (h) los astilleros son escasos.

La importancia de la industria de construcción naval de bajo bordo radica en el potencial crecimiento que otorga a la economía nacional a través de los beneficios generados por los astilleros mediante el incremento de la inversión privada, la creación de nuevos empleos y el efecto multiplicador en otras industrias conexas.

Según Moreno (2012) las construcciones de bajo bordo están comprendidos entre los 350 TPM1 hasta 2,000 TPM, las cuales pueden tener la siguiente clasificación: (a) remolcadores, (b) pesqueros, (c) barcazas, (d) chatas, (e) pontones, (f) lanchas, (g) patrulleras, y (h) cañoneras. De acuerdo al uso destinado, las embarcaciones de bajo bordo, son de los siguientes tipos: (a) embarcaciones fluviales, remolcadores de empuje según su tipo de propulsión o hélice, (c) gánguiles y pontonas, (d) corbetas, (e) fragatas $y(f)$ pesqueros.

\section{CONCLUSIONES}

La industria de la construcción naval a nivel mundial es una industria que históricamente se ha desarrollado en países con una ubicación geográfica estratégica. El Perú se encuentra ubicado en la costa oeste del Pacífico Sur y posee una larga costa que le otorga una ventaja comparativa en relación a otros países de Sudamérica.

El crecimiento a nivel mundial del comercio ha generado el crecimiento del tráfico marítimo mundial de naves de alto bordo que trasladan mercancías diversas; entre los principales tipos de naves de alto bordo se encuentran los portacontenedores, graneleros, carga general y buques tanque. Este flujo comercial marítimo genera el ámbito de desarrollo de la industria de construcción naval de bajo bordo, pues son las embarcaciones de bajo bordo las que brindan la asistencia requerida a las embarcaciones de alto bordo. La integración Perú - Brasil, la consolidación del Callao como puerto hub junto con las concesiones portuarias y de hidrovías y las potenciales oportunidades del sector pesquero permitirán la reactivación de la industria naval de bajo bordo.

La industria de construcción naval de bajo bordo nacional es de alto valor agregado y cuenta con experiencia y reconocimiento internacional, sin embargo es escaza en infraestructura moderna, tecnología de punta y mano de obra calificada. La importancia de la construcción naval reside en los beneficios económicos y sociales que esta actividad genera al país, contribuyendo a mejorar el intercambio comercial y las comunicaciones, promoviendo el empleo, la inversión, la generación de divisas, y la creación de industrias conexas.

\section{REFERENCIAS BIBLIOGRÁFICAS}

Advanced Logistics Group. (2011). Plan de Desarrollo de los Servicios de Logística de Transporte.

Agencia Andina, 2012.

Agencia Peruana de Noticias. (2012, 4 de julio). Hidrovías en Amazonía peruana podrían empezar a operar en dos años. América Economía.

Autoridad Portuaria Nacional. APN (2012). Reporte Anual 2012.

Comisión Interamericana del Atún Tropical (CIAT).

Diario Gestión, 2011.

Iniciativa para la integración de la infraestructura regional sudamericana (IIRSA). (2012). Fichas de Justificación de los criterios de selección de los proyectos de la API.

Instituto de Estudios Históricos Marítimos del Perú, IEHMP. (2012). Separata Comercio Marítimo.

Moreno, J. (2012, Diciembre). Exposición actualidad del SIMA. Charla en las instalaciones del SIMACallao, Callao, Perú

Sánchez, R. (2004). Puertos y transporte marítimo en América Latina y el Caribe: un análisis de su desempeño reciente. CEPAL: Series recursos naturales e infraestructura.

Servicios Industriales de la Marina, SIMA. (2012). Historia del SIMA. 